\title{
A New Nonrelativistic Investigation for Spectra of Heavy Quarkonia with Modified Cornell Potential: Noncommutative Three Dimensional Space and Phase Space Solutions
}

\author{
Abdelmadjid Maireche*, Djenaoui Imane ${ }^{\dagger}$ \\ Laboratory of Physics and Material Chemistry, Physics Department, University of M'sila-M'sila, Algeria
}

(Received 08 June 2016; published online 03 October 2016)

\begin{abstract}
In this paper, we present a further investigation for the exact solvability of non-relativistic quantum spectrum systems for modified Cornell potential (m.c.p.) by means Boopp's shift method instead to solving deformed Schrödinger equation (d.s.e.) with star product, in the framework of both noncommutativity three dimensional real space and space phase (NC: 3D-RSP). The exact corrections for lowest excitations states: ground and first excited states are found straightforwardly for interactions for quarkouniom systems ( $q \bar{q}$ with $q=c, b, .$. ) by means of the standard perturbation theory. Furthermore, the obtained corrections of energies are depended on: four infinitesimals parameters $(\Theta, \bar{\theta}, \chi, \bar{\sigma})$, which are induced by position-position and momentum-momentum noncommutativity, the Cornell potential parameters $(\delta, a, b)$ and the discreet atomic quantum numbers: $(j, l, s$ and $m$ ) and we have also shown that, the usual states in ordinary three dimensional spaces are canceled and has been replaced by new degenerated $2 N(2 l+1)$ sub-states in the new quantum symmetries of (NC: $3 D$-RSP). It is shown that the (d.s.e.) for (m.c.p.) has the similar behaviors to the relativistic Dirac equation which the polarities of fermionic particle appear exciplicitly.
\end{abstract}

Keywords: Cornell potential, Noncommutative space and phase, Star product and Boopp's shift method.

DOI: $10.21272 /$ jnep.8(3).03025

PACS numbers: 11.10.Nx, $32.30-\mathrm{r}, 03.65-\mathrm{w}$

\section{INTRODUCTION}

One of the important issues of modern nonrelativistic and relativistic quantum mechanics is to solve three fundamental equations: Schrödinger (for fermionic particle with $\operatorname{spin} s=1 / 2$ ), Klein-Gordon (for bosonic particle with spin zero) and Dirac (for fermionic particle with spin $r$ ) for central and non central potentials of physical and chemical interest. Although Schrödinger it is the first fundamental equation it remains interesting in the microscopic scales, it is playing a crucial role in devising well-behaved physical models in different fields of physics and chemists, many potentials are treated within the framework of nonrelativistic quantum mechanics based on this equation in two, three and D generalized spaces [1-29], the quantum structure based to the ordinary canonical commutations relations (CCRs) in both Schrödinger and Heisenberg (the operators are depended on time) pictures (CCRs), respectively, as:

$$
\begin{aligned}
& {\left[x_{i}, p_{j}\right]=i \delta_{i j} \text { and }\left[x_{i}, x_{j}\right]=\left[p_{i}, p_{j}\right]=0} \\
& {\left[x_{i}(t), p_{j}(t)\right]=i \delta_{i j} \text { and }\left[x_{i}(t), x_{j}(t)\right]=} \\
& =\left[p_{i}(t), p_{j}(t)\right]=0
\end{aligned}
$$

Where the two operators $\left(x_{i}(t), p_{i}(t)\right)$ in Heisenberg picture are related to the corresponding two operators $\left(x_{i}, p_{i}\right)$ in Schrödinger picture from the two projections relations:

$$
\begin{aligned}
& x_{i}(t)=\exp \left(i \hat{H}\left(t-t_{0}\right)\right) x_{i} \exp \left(-i \hat{H}\left(t-t_{0}\right)\right) \\
& p_{i}(t)=\exp \left(i \hat{H}\left(t-t_{0}\right)\right) p_{i} \exp \left(-i \hat{H}\left(t-t_{0}\right)\right)
\end{aligned}
$$

Here $\hat{H}$ denote to the ordinary quantum Hamiltonian operator. Recently, much considerable effort has been expanded on the solutions of Schrödinger, Dirac and Klein-Gordon equations to noncommutative quantum mechanics, the present paper investigates first the present new quantum structure which introduced firstly by H. Snyder, The Quantization of space time [30]. This new structure of quantum mechanics based to new noncommutative canonical commutations relations (NNCCRs) in both Schrödinger and Heisenberg pictures, respectively, as follows [32-59]:

$$
\begin{aligned}
& {\left[\hat{x}_{i}^{*}, \hat{p}_{j}\right]=i \delta_{i j},\left[\hat{x}_{i}^{*}, \hat{x}_{j}\right]=i \theta_{i j} \text { and }\left[\hat{p}_{i}^{*}, \hat{p}_{j}\right]=i \bar{\theta}_{i j}} \\
& {\left[\hat{x}_{i}(t), \hat{p}_{j}(t)\right]=i \delta_{i j},\left[\hat{x}_{i}(t)^{*}, \hat{x}_{j}(t)\right]=i \theta_{i j} \text { and }} \\
& {\left[\hat{p}_{i}(t)^{*}, \hat{p}_{j}(t)\right]=i \bar{\theta}_{i j}}
\end{aligned}
$$

Where the two new operators in $\left(\hat{x}_{i}(t), \hat{p}_{i}(t)\right)$ Heisenberg picture are related to the corresponding two new operators $\left(\hat{x}_{i}, \hat{p}_{i}\right)$ in Schrödinger picture from the two projections relations in (NC: $3 D-R S P)$ symmetries:

$$
\begin{aligned}
& \hat{x}_{i}(t)=\exp \left(i \hat{H}_{n c-c p}\left(t-t_{0}\right)\right) * \hat{x}_{i} * \exp \left(-i \hat{H}_{n c-c p}\left(t-t_{0}\right)\right) \\
& \hat{p}_{i}(t)=\exp \left(i \hat{H}_{n c-c p}\left(t-t_{0}\right)\right) * \hat{p}_{i} * \exp \left(-i \hat{H}_{n c-c p}\left(t-t_{0}\right)\right)
\end{aligned}
$$

\footnotetext{
* abmaireche@gmail.com

$\dagger$ djenaoui2834@gmail.com
} 
Here $\hat{H}_{n c-c p}$ denote to the new quantum Hamiltonian operator in the symmetries of (NC: $3 D-R S P)$. The very small two parameters $\theta^{\mu \nu}$ and $\bar{\theta}^{\mu \nu}$ (compared to the energy) are elements of two antisymmetric real matrixes and $(*)$ denote to the new star product, which is generalized between two arbitrary functions $f(x, p)$ and $g(x, p)$ to $(f * g)(x, p)$ instead of the usual product $(f g)(x, p)$ in ordinary three dimensional spaces [39-57]:

$$
\begin{aligned}
& (f * g)(x, p) \equiv \exp \left(\frac{i}{2} \theta^{\mu v} \partial_{\mu}^{x} \partial_{v}^{x}+\frac{i}{2} \bar{\theta}^{\mu v} \partial_{\mu}^{p} \partial_{v}^{p}\right)(f g)(x, p) \\
& =(f g)(x, p)+\left.\left(-\frac{i}{2} \theta^{\mu v} \partial_{\mu}^{x} f \partial_{\nu}^{x} g-\frac{i}{2} \bar{\theta}^{\mu v} \partial_{\mu}^{p} f \partial_{\nu}^{p} g\right)(x, p)\right|_{\left(x^{\mu}=x^{v}, p^{\mu}=p^{\nu}\right)} \\
& +O\left(\theta^{2}, \bar{\theta}^{2}\right)
\end{aligned}
$$

Where the two covariant derivatives $\left(\partial_{\mu}^{x} f(x, p), \partial_{\mu}^{p} f(x, p)\right) \quad$ are denotes to the $\left(\frac{\partial f(x, p)}{\partial x^{\mu}}, \frac{\partial f(x, p)}{\partial p^{\mu}}\right)$, respectively, and the two following terms $\quad\left(-\frac{i}{2} \theta^{\mu v} \partial_{\mu}^{x} f(x, p) \partial_{v}^{x} g(x, p)\right) \quad$ and $\left(-\frac{i}{2} \bar{\theta}^{\mu v} \partial_{\mu}^{p} f(x, p) \partial_{\nu}^{p} g(x, p)\right)$ are induced by (space-space) and (phase-phase) noncommutativity properties, respectively, $O\left(\theta^{2}, \bar{\theta}^{2}\right)$ stands for the second and higher order terms of $\theta$ and $\bar{\theta}$, a Boopp's shift method can be used, instead of solving any quantum systems by using directly star product procedure [39-55]:

$$
\left[\hat{x}_{i}, \hat{x}_{j}\right]=i \theta_{i j} \text { and }\left[\hat{p}_{i}, \hat{p}_{j}\right]=i \bar{\theta}_{i j}
$$

We can map commutative space-commutative space phase onto noncommutative space-noncommutative space phase, via Bopp shifts, so the generalized coordinates $(x, y, z)$ and momentums $\left(p_{x}, p_{y}, p_{z}\right)$ changers to new generalized coordinates $(\hat{x}, \hat{y}, \hat{z})$ and new generalized momentums $\left(\hat{p}_{x}, \hat{p}_{y}, \hat{p}_{z}\right)$, respectively, as follows [32-55]:

$$
\left\{\begin{array}{l}
x \rightarrow \hat{x}=x-\frac{\theta_{12}}{2} p_{y}-\frac{\theta_{13}}{2} p_{z} \\
y \rightarrow \hat{y}=y-\frac{\theta_{21}}{2} p_{x}-\frac{\theta_{23}}{2} p_{z} \\
z \rightarrow \hat{z}=z-\frac{\theta_{31}}{2} p_{x}-\frac{\theta_{32}}{2} p_{y}
\end{array}\right.
$$

and

$$
\left\{\begin{array}{l}
p_{x} \rightarrow \hat{p}_{x}=p_{x}-\frac{\bar{\theta}_{12}}{2} y-\frac{\bar{\theta}_{13}}{2} z \\
p_{y} \rightarrow \hat{p}_{y}=p_{y}-\frac{\bar{\theta}_{21}}{2} x-\frac{\bar{\theta}_{23}}{2} z \\
p_{z} \rightarrow \hat{p}_{z}=p_{z}-\frac{\bar{\theta}_{31}}{2} x-\frac{\bar{\theta}_{32}}{2} y
\end{array}\right.
$$

The non-vanish 9-commutators in (NC-3D: $R S P)$ can be determined as follows:

$$
\begin{aligned}
& {\left[\hat{x}, \hat{p}_{x}\right]=\left[\hat{y}, \hat{p}_{y}\right]=\left[\hat{z}, \hat{p}_{z}\right]=i,} \\
& {[\hat{x}, \hat{y}]=i \theta_{12},[\hat{x}, \hat{z}]=i \theta_{13},[\hat{y}, \hat{z}]=i \theta_{23}} \\
& {\left[\hat{p}_{x}, \hat{p}_{y}\right]=i \bar{\theta}_{12},\left[\hat{p}_{y}, \hat{p}_{z}\right]=i \bar{\theta}_{23},\left[\hat{p}_{x}, \hat{p}_{z}\right]=i \bar{\theta}_{13}}
\end{aligned}
$$

After a straightforward calculations, using eqs. (3.2) and (3.3), we can show that the two operators $\hat{r}^{2}$ and $\hat{p}^{2}$ on a noncommutative three dimensional spaces and space phase are determined with two equations, respectively [32-48]:

$$
\hat{r}^{2}=r^{2}-\overrightarrow{\mathbf{L}} \vec{\Theta} \quad \text { and } \quad \hat{p}^{2}=p^{2}+\overrightarrow{\mathbf{L}} \overrightarrow{\boldsymbol{\theta}}
$$

Where the two couplings $\mathbf{L} \Theta\left(\Theta \equiv \frac{\theta}{2}\right)$ and $\overrightarrow{\mathbf{L}} \overrightarrow{\vec{\theta}}$ are given by, respectively:

$$
\begin{gathered}
\mathbf{L} \Theta \equiv L_{x} \Theta_{12}+L_{y} \Theta_{23}+L_{z} \Theta_{13} \quad \text { and } \\
\overrightarrow{\mathbf{L}} \overrightarrow{\overline{\boldsymbol{\theta}}} \equiv L_{x} \bar{\theta}_{12}+L_{y} \bar{\theta}_{23}+L_{z} \bar{\theta}_{13}
\end{gathered}
$$

It is-well known, that, in usual quantum mechanics, the three components $\left(L_{x}, L_{y}\right.$ and $L_{z}$ ) are determined, in spherical coordinates $(r, \theta, \phi)$ as follows:

$$
\begin{aligned}
L_{x} & =i\left(\sin \theta \frac{\partial}{\partial \theta}+\frac{\cos \phi}{\operatorname{tg} \theta} \frac{\partial}{\partial \phi}\right), \\
L_{y} & =-i\left(-\cos \phi \frac{\partial}{\partial \theta}+\frac{\sin \phi}{\operatorname{tg} \theta} \frac{\partial}{\partial \phi}\right) \\
& \text { and } L_{z}=\frac{1}{i} \frac{\partial}{\partial \phi}
\end{aligned}
$$

The study of modified Cornell potential has now become a very interest field due to their applications in different fields, in modeling the interaction potentials of the quark-antiquark systems typically use hold-type potentials and it is very urgent to study the potential quark for lattice QCD and QCD sum rules [30], here, we shall attempt to solve the (d.s.e.) in $(N C$ : $3 D-R S P)$ by using the generalization of Boopp's shift method, which is a direct results of star product and recently used by many authors to solve (d.s.e.) for different potential forms [32-57]. It is important to notice that, the author A. Al-Jamel has been studied the potential $V(r)=k r-\frac{4}{3} \frac{\alpha_{s}}{r}$ on noncommutative space, to the second order in to the second order in $\theta$ [31], our work is aimed to extending this study to include the noncommutativity of space phase, but in the two infinitesimals parame$\operatorname{ters}(\Theta, \bar{\theta})$ to discover the new symmetries and a possibility to obtain other applications to this potential in other fields. The organization scheme of the study is given as follows: In next section, we briefly review the Schrödinger equation with Cornell potential on based to Ref. [29]. The Section 3, devoted to studying the three deformed Schrödinger equation by applying both Boopp's shift method to the Cornell potential. In the 
fourth section and by applying standard perturbation theory we find the quantum spectrum of the excited states in (NC-3D: RSP) for spin-orbital interaction. In the next section, we derive the magnetic spectrum for (m.c.p.). In the sixth section, we resume the global spectrum and corresponding noncommutative Hamiltonian for (m.c.p.). Finally, the important results and the conclusions are discussed in last section.

\section{REVIEW THE EIGNENFUNCTIONS AND THE ENERGY EIGENVALUES FOR CORNELL POTENTIAL IN ORDINARY THREE DIMEN- SIONAL SPACES}

As it is studied, in ref [29], the interaction potential between a quark and anti-quark is determined from the following central potential, namely Cornell potential:

$$
V(r)=a r-\frac{b}{r}
$$

where $a$ and $b$ are two positive constant coefficients and $r$ is the interquark distance, this systems known by Quarkoniums systems $(c \bar{c}, b \bar{b}, c \bar{b}, b \bar{c}, \ldots$.$) . The above$ potential is the sum of linear part (ar) which account for quark confinement at large distances and the second part $\left(-\frac{b}{r}\right)$ induced by one gluon exchange between the quarks ant it is anti-quark in short distances. The radial function $R_{l}(r)$ satisfied the following equation, in three dimensional spaces [29]:

$$
\begin{aligned}
& \frac{1}{r^{2}} \frac{d}{d r}\left(r^{2} \frac{d R_{n l}(r)}{d r}\right)+ \\
& +\left[2 \mu(\mathrm{E}-V(r))-\frac{l(l+1)}{r^{2}}\right] R_{n l}(r)=0
\end{aligned}
$$

Here $\mu=\frac{m_{q} m_{\bar{q}}}{m_{q}+m_{\bar{q}}} \quad$ is the reduced of quarkonium systems composed with the rest mass of quark and anti-quark ( $m_{q}$ and $m_{\bar{q}}$ ), respectively. The reduced radial wave functions: $X_{n l}(r)=r R_{n l}(r)$ is introduced to obtain the following second order differential equation:

$$
X_{n l}^{\prime \prime}(r)+\left[2 \mu(E-V(r))-\frac{l(l+1)}{r^{2}}\right] X_{n l}(r)
$$

The Nikivarov-Uvarov method has been applied in ref. [29] to obtain the radial wave equation and corresponding eigenvalues energy:

$$
R_{n l}(r)=N_{n l} r^{-\frac{B}{\sqrt{2 A}}-1} e^{\sqrt{2 A} r}\left(-r^{2} \frac{d}{d r}\right)^{n}\left(r^{-2 n+\frac{2 B}{\sqrt{2 A}}} e^{-2 \sqrt{2 A} r}\right)
$$

and

$$
E_{n, l}=\frac{3 a}{\delta}-\frac{2 \mu\left(b+\frac{3 a}{\delta^{2}}\right)^{2}}{\left[(2 n+1) \pm \sqrt{1+4 l(l+1)+\frac{8 \mu}{\delta^{3}}}\right]^{2}}
$$

with $A=-\mu\left(E-\frac{3 a}{\delta}\right), B=\mu\left(b+\frac{3 a}{\delta^{2}}\right)$ and $\delta=\frac{1}{r_{0}}, r_{0}$ is a characteristic radius of the meson. For ground state and first excited states, we can deduce easily after a simple calculations the following solutions:

$$
\begin{aligned}
& \psi^{(0)}(\vec{r})=N_{0 l} r^{\frac{B}{\sqrt{2 A}}-2} e^{-2 \sqrt{2 A} r} Y_{l}^{m}(\theta, \phi) \\
& \text { and } E_{0 l}=\frac{3 a}{\delta}-\frac{2 \mu\left(b+\frac{3 a}{\delta^{2}}\right)^{2}}{\left[ \pm \sqrt{1+4 l(l+1)+\frac{8 \mu a}{\delta^{3}}}\right]^{2}}
\end{aligned}
$$

and

$$
\begin{aligned}
& \psi^{(1)}(\vec{r})=N_{1 l}\left(-\eta r^{\frac{B}{\sqrt{2 A}}-3}+\sigma r^{\frac{B}{\sqrt{2 A}}-1}\right) e^{-2 \sqrt{2 A} r} Y_{l}^{m}(\theta, \phi) \\
& \text { and } E_{1 l}=\frac{3 a}{\delta}-\frac{2 \mu\left(b+\frac{3 a}{\delta^{2}}\right)^{2}}{\left[3 \pm \sqrt{1+4 l(l+1)+\frac{8 \mu a}{\delta^{3}}}\right]^{2}}
\end{aligned}
$$

Where $\eta$ and $\sigma$ are given by:

$$
\eta=-2+\frac{2 B}{\sqrt{2 A}} \text { and } \sigma=2 \sqrt{2 A}
$$

\section{DEFORMED SCHRÖDINGER EQUATION AND MODIFIED CORNELL POTENTIAL IN (NC-3D: RSP):}

This section is devoted to constructing of non relativistic modified Schrödinger equations in (NC-3D: $R S P$ ) for (m.c.p.); to achieve this subject, we apply the essentials following steps [32-48]:

- Ordinary three dimensional Hamiltonian operator $\hat{H}_{c p}\left(p_{i}, x_{i}\right)$ will be replaced by new three dimensional Hamiltonian operator $\hat{H}_{n c-c p}\left(\hat{p}_{i}, \hat{x}_{i}\right)$,

- Ordinary complex wave function $\Psi(\vec{r})$ will be replacing by new complex wave function $\widehat{\Psi}(\overrightarrow{\vec{r}})$,

- Ordinary energies $E_{n, l}$ will be replaced by new values $E_{n c-c p}$,

And the last step corresponds to replace the ordinary old product by new star product $(*)$, which allow us to constructing the deformed three dimensional Schrödinger equation in (NC-3D: RSP) as for (m.c.p.) potential:

$$
\hat{H}_{n c-c p}\left(\hat{p}_{i}, \hat{x}_{i}\right) * \widehat{\Psi}(\overrightarrow{\hat{r}})=E_{n c-c p} \hat{\Psi}(\overrightarrow{\vec{r}})
$$

In order to use the ordinary product without star 
product, with new vision, as mentioned before, we apply the Boopp's shift method on the above eq. (13) to obtain the reduced Schrödinger in $(N C-3 D: R S P)$ for (m.c.p.):

$$
H_{n c-c p}\left(\hat{p}_{i}, \hat{x}_{i}\right) \psi(\vec{r})=E_{n c-c p} \psi(\vec{r})
$$

Where the new operator of Hamiltonian $H_{n c-c p}\left(\hat{p}_{i}, \hat{x}_{i}\right)$ can be expressed in three general noncommutative quantum varieties: both noncommutative space and noncommutative phase ( $N C-3 D: R S P)$, only noncommutative space $(N C-3 D: R S)$ and only noncommutative phase $(N C: 3 D-R P)$ as, respectively:

$$
H_{n c-c p}\left(\hat{p}_{i}, \hat{x}_{i}\right) \equiv H\left(p_{x}+\frac{\bar{\theta}}{2} y, p_{y}-\frac{\bar{\theta}}{2} x, x-\frac{\theta}{2} p_{y}, y+\frac{\theta}{2} p_{x}\right)
$$

for NC-3D: RSP

$$
\begin{aligned}
& H_{n c-c p}\left(\hat{p}_{i}, \hat{x}_{i}\right) \equiv H\left(p_{x}, p_{y}, x-\frac{\theta}{2} p_{y}, y+\frac{\theta}{2} p_{x}\right) \\
& \text { for } \mathrm{NC}-3 \mathrm{D}: \mathrm{RS} \\
& H_{n c-c p}\left(\hat{p}_{i}, \hat{x}_{i}\right) \equiv H\left(p_{x}+\frac{\bar{\theta}}{2} y, p_{x}-\frac{\bar{\theta}}{2} x, x, y\right) \\
& \text { for } \mathrm{NC}-3 \mathrm{D}: \mathrm{RP}
\end{aligned}
$$

In recently work, we are interest with the first variety (15.1), after straightforward calculations, we can obtain the three important terms, which will be use to determine the (m.c.p.) potential in (NC: 3D-RSP), respectively, as:

$$
\begin{aligned}
& a \hat{r}=a r-a \frac{\overrightarrow{\mathbf{L}} \vec{\Theta}}{2 r}, \quad \frac{b}{\hat{r}}=\frac{b}{r}+b \frac{\overrightarrow{\mathbf{L}} \vec{\Theta}}{2 r^{3}} \quad \text { and } \\
& \frac{\hat{p}^{2}}{2 m_{0}}=\frac{p^{2}}{2 m_{0}}+\frac{\overrightarrow{\mathbf{L}} \boldsymbol{\theta}}{2 m_{0}}
\end{aligned}
$$

Which allow us to obtaining the global potential operator $H_{n c-c p}\left(\hat{p}_{i}, \hat{x}_{i}\right)$ for (m.c.p.) in (NC: $\left.3 D-R S P\right)$, respectively, as:

$$
\begin{aligned}
& H_{n c-c p}\left(\hat{p}_{\mathrm{i}}=p_{i}-\frac{\bar{\theta}^{\mathrm{ij}}}{2} x_{j}, \hat{x}_{\mathrm{i}}=x_{i}-\frac{\theta^{\mathrm{ij}}}{2} p_{j}\right)= \\
& =a r-\frac{b}{r}+\frac{p^{2}}{2 m_{0}}+\left(\frac{b}{2 r^{3}}-\frac{a}{2 r}\right) \vec{L} \vec{\Theta}+\frac{\vec{L} \overrightarrow{\bar{\theta}}}{2 \mu}
\end{aligned}
$$

It's clearly, that the three first terms are given the ordinary Cornell potential and kinetic energy in three dimensional spaces while the rest terms are proportional's with infinitesimals parameters $(\Theta, \bar{\theta})$, thus, we can considered as a perturbations term, we noted by $\hat{H}_{c p-p e r t}(r, a, b, \Theta, \bar{\theta})$ for (NC: 3D-RSP) symmetries, respectively, as:

$$
\hat{H}_{c p-p e r t}(r, a, b, \Theta, \bar{\theta})=\left(\frac{b}{2 r^{3}}-\frac{a}{2 r}\right) \vec{L} \vec{\Theta}+\frac{\vec{L} \overrightarrow{\vec{\theta}}}{2 \mu}
$$

\section{FOR (M.C.P.) IN (NC: 3D- RSP) SYMMETRIES FOR LOWEST EXCITATIONS STATES FOR QUARKONIUM SYSTEMS}

4.1 The Exact Spin-orbital Hamiltonian for (m.c.p.) in (NC: 3D-RSP) Symmetries for Quarkonium Systems:

Again, the perturbative two terms $\hat{H}_{c p-p e r t}(r, a, b, \Theta, \bar{\theta})$ can be rewritten to the equivalent physical form for (m.c.p.):

$$
\hat{H}_{c p-p e r t}(r, A, B, \Theta, \bar{\theta})=\left\{\frac{\bar{\theta}}{2 \mu}+\Theta\left(\frac{b}{2 r^{3}}-\frac{a}{2 r}\right)\right\} \vec{S} \vec{L}
$$

Furthermore, the above perturbative operator $\hat{H}_{c p-p e r t}(r, a, b, \Theta, \bar{\theta})$ can be rewritten to the following new equivalent form for (m.c.p.) as follows:

$$
\begin{aligned}
& \hat{H}_{c p-p e r t}(r, a, b, \Theta, \bar{\theta})=\frac{1}{2}\left\{\left\{\frac{\bar{\theta}}{2 \mu}+\Theta\left(\frac{b}{2 r^{3}}-\frac{a}{2 r}\right)\right\}\right\} \times \\
& \times\left(\overleftrightarrow{J}^{2}-\overleftrightarrow{L}^{2}-\overleftrightarrow{S}^{2}\right)
\end{aligned}
$$

To the best of our knowledge, we just replace the coupling spin-orbital $\vec{S} \overleftrightarrow{L}$ by the expres$\operatorname{sion} \frac{1}{2}\left(\vec{J}^{2}-\vec{L}^{2}-\vec{S}^{2}\right)$, in quantum mechanics. The set ( $H_{n c-c p}\left(\hat{p}_{i}, \hat{x}_{i}\right), \mathrm{J}^{2}, \mathrm{~L}^{2}, \mathrm{~S}^{2}$ and $\left.J_{z}\right)$ forms a complete of conserved physics quantities and the eigenvalues of the spin orbital coupling operator are:

$$
\vec{L} \vec{S} \Psi=\left\{\begin{array}{l}
\frac{1}{2}\left\{(l+s)\left(l+\frac{1}{2}+1\right)+l(l+1)-s(s+1)\right\} \times \\
\times \Psi \equiv p_{+} \Psi: \text { for } \quad j=|l+s| \\
\frac{1}{2}\left\{(l-s)\left(l-\frac{1}{2}+1\right)+l(l+1)-s(s+1)\right\} \times \\
\times \Psi \equiv p_{-} \Psi \text { : for } \quad j=|l-s|
\end{array}\right.
$$

Which allows us to form a diagonal $(3 \times 3)$ matrix, with non null elements are $\left[\left(\hat{H}_{s o-c p}\right)_{11},\left(\hat{H}_{s o-c p}\right)_{22},\left(\hat{H}_{s o-c p}\right)_{33}\right]$ for (m.c.p.) potential in $(N C: 3 D-R S P)$, respectively, as:

$$
\left(\hat{H}_{s o-c p}\right) \equiv\left(\begin{array}{ccc}
\left(\hat{H}_{s o-c p}\right)_{11} & 0 & 0 \\
0 & \left(\hat{H}_{s o-c p}\right)_{22} & 0 \\
0 & 0 & \left(\hat{H}_{s o-c p}\right)_{33}
\end{array}\right)
$$

Substituting two equations (19) into equation (14) and then, the radial part of the (d.s.e.), satisfying the following important equation:

\section{THE EXACT SPIN-ORBITAL HAMILTONIAN AND THE CORRESPONDING SPECTRUM}




$$
\begin{aligned}
& \frac{1}{r^{2}} \frac{d}{d r}\left(r^{2} \frac{d R_{n l}(r)}{d r}\right)+ \\
& +\left[\begin{array}{l}
2 \mu\left(\mathrm{E}_{n c-c p}-a r+\frac{b}{r}\right)-\frac{l(l+1)}{r^{2}}- \\
-\left\{\frac{\bar{\theta}}{2 \mu}+\Theta\left(\frac{A}{r^{4}}-\frac{B}{2 r^{3}}\right)\right\} \vec{S} \vec{L}
\end{array}\right] R_{n l}(r)=0
\end{aligned}
$$

It is clearly that the above equation including the perturbative term of Hamiltonian operator $\hat{H}_{c p-p e r t}(r, A, B, \Theta, \bar{\theta})$ which we are subject of discussion in next sub-section.

\subsection{The Exact Spin-orbital Spectrum for (m.c.p.) Potential in (NC: $3 D$ - RSP) Symmetries for Ground States for Quarkonium Systems}

After determining the perturbative term of Hamiltonian operator $\hat{H}_{c p-p e r t}(r, A, B, \Theta, \bar{\theta})$, it is possible to apply the standard perturbation theory to obtains the nonrelativistic modifications to the energy levels $E_{n c 0-p e r}(\Theta, \bar{\theta}, a, b, \delta)$ for quarkonium systems, at first order of two parameters $(\Theta, \bar{\theta})$, for ground states, using eqs. (11) and (20) corresponding (NC-3D: RSP), respectively, as:

$$
\begin{aligned}
& E_{n c 0-p e r}(\Theta, \bar{\theta}, a, b, \delta)= \\
& =\frac{1}{2} \alpha\left|N_{0 k}\right|^{2}\{j(j+1)+l(l+1)-s(s+1)\} \\
& \int r^{\frac{2 B}{\sqrt{2 A}}-2} e^{-2 \sqrt{2 A} r}\left[\Theta\left(\frac{b}{2 r^{3}}-\frac{a}{2 r}\right)+\frac{\bar{\theta}}{2 m_{0}}\right] d r
\end{aligned}
$$

A direct simplification gives:

$$
\begin{aligned}
& E_{n c 0-p e r}(\Theta, \bar{\theta}, a, b, \delta)= \\
& =\frac{1}{2} \alpha\left|N_{0 k}\right|^{2}\{j(j+1)+l(l+1)-s(s+1)\} \\
& \left(\Theta \sum_{i=1}^{2} T_{i}(a, b, \delta)+\frac{\bar{\theta}}{2 m_{0}} T_{3}(a, b, \delta)\right)
\end{aligned}
$$

Where, the 3- terms: $T_{i}(a, b, \delta)$ are given by:

$$
\begin{aligned}
& T_{1}(a, b, \delta)=\frac{b}{2} \int_{0}^{+\infty} r^{\frac{2 B}{\sqrt{2 A}}-5} e^{-2 \sqrt{2 A} r} d r \\
& T_{2}(a, b, \delta)=-\frac{a}{2} \int_{0}^{+\infty} r^{\frac{2 B}{\sqrt{2 A}}-3} e^{-2 \sqrt{2 A} r} d r \\
& T_{3}(a, b, \delta)=\int_{0}^{+\infty} r^{\frac{2 B}{\sqrt{2 A}}-2} e^{-2 \sqrt{2 A} r} d r
\end{aligned}
$$

Know we apply the special integral [60]:

$$
\int_{0}^{+\infty} x^{m} \exp \left(-\beta x^{n}\right) d x=\frac{\Gamma\left(\frac{m+1}{n}, \beta x^{n}\right)}{n \beta^{\frac{m+1}{n}}}
$$

$\Gamma\left(\frac{m+1}{n}, \beta x^{n}\right)$ Is the incomplete Gamma function, the three values of $(m, n, \beta)$ are determined $\left(m=\frac{2 B}{\sqrt{2 A}}-5, n=1\right.$ and $\left.\beta=2 \sqrt{2 A}\right), \quad\left(m=\frac{2 B}{\sqrt{2 A}}-3, n=1\right.$ and $\left.\beta=2 \sqrt{2 A}\right)$ and $\left(m=\frac{2 B}{\sqrt{2 A}}-2, \quad n=1\right.$ and $\left.\beta=2 \sqrt{2 A}\right)$ for $T_{i}(a, b, \delta)$ which allow us to obtaining :

$$
\begin{gathered}
T_{1}(a, b, \delta)=\frac{b}{2} \frac{\Gamma\left(\frac{2 B}{\sqrt{2 A}}-4,2 \sqrt{2 A} r\right)}{(2 \sqrt{2 A}) \frac{2 B}{\sqrt{2 A}}-4} \\
T_{2}(a, b, \delta)=-\frac{a}{2} \frac{\Gamma\left(\frac{2 B}{\sqrt{2 A}}-2,2 \sqrt{2 A} r\right)}{(2 \sqrt{2 A}) \frac{2 B}{\sqrt{2 A}}-2} \\
T_{3}(a, b, \delta)=\frac{\Gamma\left(\frac{2 B}{\sqrt{2 A}}-1,2 \sqrt{2 A} r\right)}{(2 \sqrt{2 A}) \frac{2 B}{\sqrt{2 A}}-1}
\end{gathered}
$$

Which allow us to obtaining the exact modifications of ground states $E_{n c 0-p e r}(\Theta, \bar{\theta}, a, b, \delta)$ as follows:

$$
\begin{aligned}
& E_{n c 0-p e r}(\Theta, \bar{\theta}, a, b, \delta)=\frac{1}{2} \alpha\left|N_{0 k}\right|^{2}\{j(j+1)+l(l+1)-s(s+1)\} \\
& \left(\Theta L_{o}(a, b, \delta)+\frac{\bar{\theta}}{2 m_{0}} T_{3}(a, b, \delta)\right)
\end{aligned}
$$

Where, the new factor $L_{o}(a, b, \delta)$ is given by:

$$
\begin{aligned}
& L_{o}(a, b, \delta)=\frac{b}{2} \frac{\Gamma\left(\frac{2 B}{\sqrt{2 A}}-4,2 \sqrt{2 A} r\right)}{(2 \sqrt{2 A})^{\frac{2 B}{\sqrt{2 A}}}-4}- \\
& -\frac{a}{2} \frac{\Gamma\left(\frac{2 B}{\sqrt{2 A}}-2,2 \sqrt{2 A} r\right)}{(2 \sqrt{2 A})^{\frac{2 B}{\sqrt{2 A}}-2}}
\end{aligned}
$$

4.3 The Exact Spin-orbital Spectrum for (m.c.p.) Potential in (NC: 3D- RSP) Symmetries for First Excited States for Quarkouniom Systems

Know, we are going to study the nonrelativistic modifications to the energy levels $E_{n c 1-p e r}(\Theta, \bar{\theta}, a, b, \delta)$ for quarkonium systems, at first order of parameters $(\Theta, \bar{\theta})$, for first excited states, using two eqs. (12.1) and (20), to obtain:

$$
\begin{aligned}
& E_{n c 1-p e r}(\Theta, \bar{\theta})=\frac{1}{2} \alpha\left|N_{1 k}\right|^{2}\{j(j+1)+l(l+1)-s(s+1)\} \\
& \int_{0}^{+\infty}\left(-\eta^{2} r^{\frac{2 B}{\sqrt{2 A}}-6}+\sigma^{2} r^{\frac{2 B}{\sqrt{2 A}}-2}-2 \eta \sigma r^{\frac{2 B}{\sqrt{2 A}}-4}\right) e^{-2 \sqrt{2 A} r} \times \\
& \times\left(\frac{b}{2 r^{3}}-\frac{a}{2 r}+\frac{\bar{\theta}}{2 m_{0}}\right) d r
\end{aligned}
$$


a direct simplification gives $E_{n c 1-p e r}(\Theta, \bar{\theta},, a, b, \delta)$ s a function of new factors $L_{i},(a, b, \delta)$, as:

$$
\begin{aligned}
& E_{n c 1-p e r}(\Theta, \bar{\theta}, a, b, \delta)= \\
& =\frac{1}{2} \alpha\left|N_{1 k}\right|^{2}\{j(j+1)+l(l+1)-s(s+1)\} \\
& \left(\Theta \sum_{i=1}^{6} L_{i}(a, b, \delta)+\frac{\bar{\theta}}{2 \mu} \sum_{i=7}^{9} L_{i}(a, b, \delta)\right)
\end{aligned}
$$

Where, the nine terms $T_{i}(a, b, \delta)$ are determined from:

$$
\begin{aligned}
& L_{1}(a, b, \delta)=-\frac{b}{2} \eta^{2} \int_{0}^{+\infty} r^{\frac{2 B}{\sqrt{2 A}}-9} e^{-2 \sqrt{2 A} r} d r \\
& L_{2}(a, b, \delta)=-\frac{a}{2} \eta^{2} \int_{0}^{+\infty} r^{\frac{2 B}{\sqrt{2 A}}-7} e^{-2 \sqrt{2 A} r} d r \\
& V_{3}(a, b, \delta)=\frac{b}{2} \sigma^{2} \int_{0}^{+\infty} r^{\frac{2 B}{\sqrt{2 A}}-5} e^{-2 \sqrt{2 A} r} d r \\
& L_{4}(a, b, \delta)=-\frac{a}{2} \sigma^{2} \int_{0}^{+\infty} \frac{2 B}{\sqrt{2 A}}-3 e^{-2 \sqrt{2 A} r} d r \\
& L_{5}(a, b, \delta)=-b \eta \sigma \int_{0}^{+\infty} \frac{2 B}{\sqrt{2 A}}-7 e^{-2 \sqrt{2 A} r} d r \\
& L_{6}(a, b, \delta)=-a \eta \sigma \int_{0}^{+\infty} r^{\frac{2 B}{\sqrt{2 A}}-5} e^{-2 \sqrt{2 A} r} d r \\
& L_{7}(a, b, \delta)=\eta^{2} \int_{0}^{+\infty} r^{\frac{2 B}{\sqrt{2 A}}-6} e^{-2 \sqrt{2 A} r} d r \\
& L_{8}(a, b, \delta)=\sigma^{2} \int_{0}^{+\infty} r^{\frac{2 B}{\sqrt{2 A}}-2} e^{-2 \sqrt{2 A} r} d r \\
& L_{9}(a, b, \delta)=-2 \eta \sigma \int_{0}^{+\infty} r^{\frac{2 B}{\sqrt{2 A}}-4} e^{-2 \sqrt{2 A} r} d r
\end{aligned}
$$

The three values of $(m, n, \beta)$ which appears in special integral (27) for 9- terms $T_{i}(a, b, \delta)$ are determined from the explicitly relations:

$$
\begin{aligned}
& \left(m=\frac{2 B}{\sqrt{2 A}}-9, \quad n=1 \text { and } \beta=2 \sqrt{2 A}\right) \\
& \left(m=\frac{2 B}{\sqrt{2 A}}-7, \quad n=1 \text { and } \beta=2 \sqrt{2 A}\right) \\
& \left(m=\frac{2 B}{\sqrt{2 A}}-6, \quad n=1 \text { and } \beta=2 \sqrt{2 A}\right) \\
& \left(m=\frac{2 B}{\sqrt{2 A}}-5, \quad n=1 \text { and } \beta=2 \sqrt{2 A}\right) \\
& \left(m=\frac{2 B}{\sqrt{2 A}}-3, \quad n=1 \text { and } \beta=2 \sqrt{2 A}\right) \\
& \left(m=\frac{2 B}{\sqrt{2 A}}-2, \quad n=1 \text { and } \beta=2 \sqrt{2 A}\right)
\end{aligned}
$$

$$
\begin{array}{r}
\left(m=\frac{2 B}{\sqrt{2 A}}-7, \quad n=1 \text { and } \beta=2 \sqrt{2 A}\right), \\
\text { and }\left(m=\frac{2 B}{\sqrt{2 A}}-5, \quad n=1 \text { and } \beta=2 \sqrt{2 A}\right), \\
(m=4, \quad n=1 \text { and } \beta=2 \sqrt{2 A})
\end{array}
$$

Which allow us to obtaining the results of integrations as follows:

$$
\begin{aligned}
& L_{1}(a, b, \delta)=-\frac{b \eta^{2}}{2} \frac{\Gamma\left(\frac{2 B}{\sqrt{2 A}}-8,2 \sqrt{2 A} r\right)}{(2 \sqrt{2 A})^{\frac{2 B}{\sqrt{2 A}}-8}}, \\
& L_{2}(a, b, \delta)=-\frac{a \eta^{2}}{2} \frac{\Gamma\left(\frac{2 B}{\sqrt{2 A}}-6,2 \sqrt{2 A} r\right)}{(2 \sqrt{2 A})^{\frac{2 B}{\sqrt{2 A}}-6}} \text {, } \\
& L_{3}(a, b, \delta)=\frac{b \sigma^{2}}{2} \frac{\Gamma\left(\frac{2 B}{\sqrt{2 A}}-4,2 \sqrt{2 A} r\right)}{(2 \sqrt{2 A})^{\frac{2 B}{\sqrt{2 A}}-4}}, \\
& L_{4}(a, b, \delta)=\frac{b \sigma^{2}}{2} \frac{\Gamma\left(\frac{2 B}{\sqrt{2 A}}-2,2 \sqrt{2 A} r\right)}{(2 \sqrt{2 A})^{\frac{2 B}{\sqrt{2 A}}-2}}, \\
& L_{5}(a, b, \delta)=-\sigma^{2} \frac{\Gamma\left(\frac{2 B}{\sqrt{2 A}}-1,2 \sqrt{2 A} r\right)}{(2 \sqrt{2 A})^{\frac{2 B}{\sqrt{2 A}}-1}}, \\
& L_{6}(a, b, \delta)=-c \eta \sigma \frac{\Gamma\left(\frac{2 B}{\sqrt{2 A}}-6,2 \sqrt{2 A} r\right)}{(2 \sqrt{2 A})^{\frac{2 B}{\sqrt{2 A}}-6}} \text {, } \\
& T_{7}=\eta^{2} \frac{\Gamma\left(\frac{2 B}{\sqrt{2 A}}-5,2 \sqrt{2 A} r\right)}{(2 \sqrt{2 A})^{\frac{2 B}{\sqrt{2 A}}-5}}, \\
& L_{8}(a, b, \delta)=-b \eta \sigma \frac{\Gamma\left(\frac{2 B}{\sqrt{2 A}}-4,2 \sqrt{2 A} r\right)}{(2 \sqrt{2 A})^{\frac{2 B}{\sqrt{2 A}}-4}}, \\
& L_{9}(a, b, \delta)=2 \eta \sigma \frac{\Gamma\left(\frac{2 B}{\sqrt{2 A}}-3,2 \sqrt{2 A} r\right)}{(2 \sqrt{2 A})^{\frac{2 B}{\sqrt{2 A}}-3}} .
\end{aligned}
$$

It is important to notice that the terms (35.1-9) are the same terms which we obtained in our ref. [47]. Thus, the exact modifications for first excited states produced by spin-orbital effect: 


$$
\begin{aligned}
& E_{n c 1-p e r}(\Theta, \bar{\theta}, a, b, \delta)= \\
& =\frac{1}{2} \alpha\left|N_{1 k}\right|^{2}\{j(j+1)+l(l+1)-s(s+1)\} \\
& \left(\Theta L_{1-s}(a, b, \delta)+\frac{\bar{\theta}}{2 m_{0}} T_{1-p}(a, b, \delta)\right)
\end{aligned}
$$

Where, the factors $L_{1-s}(a, b, \delta)$ and $T_{1-p}(a, b, \delta)$ are given b:

$$
\begin{aligned}
& L_{1-s}(a, b, \delta)=\sum_{i=1}^{6} L_{i}(a, b, \delta) \\
& T_{1-p}(a, b, \delta)=\sum_{i=7}^{9} L_{i}(a, b, \delta)
\end{aligned}
$$

4.4 The Exact Magnetic Spectrum for (m.i.s.) Potential in (NC: 3D- RSP) Symmetries for Excited $n^{\text {th }}$ States for One-electron Atoms

Having obtained the exact modifications to the energy levels $\left(E_{n c 0-p e r}(\Theta, \bar{\theta}), E_{n c 1-p e r}(\Theta, \bar{\theta})\right.$ ) for ground states and first excited states produced with spinorbital Hamiltonian operator, we now consider new interested physically meaningful phenomena, which also produced from the perturbative term of modified Cornell potential related to the influence of an external uniform magnetic field, at first order of two parameters $(\Theta, \bar{\theta})$, it's sufficient to apply the following three replacements to describing these phenomena:

$$
\begin{aligned}
& \frac{\overrightarrow{\mathbf{L}} \overrightarrow{\boldsymbol{\theta}}}{2 \mu}+\left(\frac{b}{2 r^{3}}-\frac{a}{2 r}\right) \overrightarrow{\mathbf{L}} \vec{\Theta} \rightarrow\left\{\frac{\bar{\sigma}}{2 m_{0}}+\chi\left(\frac{b}{2 r^{3}}-\frac{a}{2 r}\right)\right\} \vec{H} \vec{L} \\
& \theta \rightarrow \chi H, \Theta \rightarrow \chi H \quad \text { and } \bar{\theta} \rightarrow \bar{\sigma} H \ldots \ldots \ldots \ldots \ldots \ldots . .
\end{aligned}
$$

Here $\chi$ and $\bar{\sigma}$ are new infinitesimal real proportional's constants, and we choose the magnetic field $\vec{H}=H \vec{k}$, which allow us to introduce the modified new magnetic Hamiltonian $\hat{H}_{m-c p}(r, A, B, \chi, \bar{\sigma})$ in (NC: 3D-RSP), as follows:

$$
\hat{H}_{m-c p}(r, A, B, \chi, \bar{\sigma})=\left(\chi\left(\frac{b}{2 r^{3}}-\frac{a}{2 r}\right)+\frac{\bar{\sigma}}{2 \mu}\right)(\vec{H} \vec{J}-\vec{S} \overleftrightarrow{H})
$$

It is well know that, the coupling between spin $\vec{S}$ and external magnetic field $\overleftrightarrow{H}$ is the ordinary Hamiltonian of Zeeman Effect in usual commutative space. So the noncommutative contributions modify the ordinary Hamiltonian of Zeeman by new coupling $\vec{H} \vec{J}$. Know, to avoid repetition in the previous solution of (28) and (33.1-3), the exact noncommutative magnetic modifications of energy $\left(E_{\text {mag0-cp }}(\chi, \bar{\sigma}, m, \delta, a, b)\right.$ and $\left.E_{\text {mag-1cp }}(\chi, \bar{\sigma}, m, \delta, a, b)\right)$ for (m.c.p.) produced automatically by the effect of $\hat{H}_{m-c p}(r, a, b, \chi, \bar{\sigma})$, can be obtained directly from those of the spin-orbital energies by using the parameter mapping:

$$
\begin{aligned}
& p \equiv j(j+1)+l(l+1)-s(s+1) \rightarrow \mathrm{m} \\
& (\bar{\theta}, \Theta) \rightarrow(\bar{\sigma}, \chi) \mathrm{H}
\end{aligned}
$$

Following the previous results (29) and (36) with the above three transformations, we finally arrive at two values ( $E_{\text {mag0-cp }}(\chi, \bar{\sigma}, m, \delta, a, b)$, $\left.E_{\text {mag-1cp }}(\chi, \bar{\sigma}, m, \delta, a, b)\right)$ for the exact magnetic modifications of spectrum corresponding ground and first excited states, at first order of two parameters $(\chi, \bar{\sigma})$ in (NC-3D: RSP), respectively, as:

$$
\begin{aligned}
& E_{\text {mag0-cp }}(\chi, \bar{\sigma}, m, \delta, a, b)= \\
& =\frac{1}{2} \alpha\left|N_{0 k}\right|^{2} m\left(\chi L_{o}(a, b, \delta)+\frac{\bar{\sigma}}{2 m_{0}} T_{3}(a, b, \delta)\right) H \\
& E_{\text {mag-1cp }}(\chi, \bar{\sigma}, m, \delta, a, b)= \\
& =\frac{1}{2} \alpha\left|N_{1 k}\right|^{2} m\left(\chi L_{1-s}(a, b, \delta)+\frac{\bar{\sigma}}{2 m_{0}} L_{1-p}(a, b, \delta)\right) H
\end{aligned}
$$

Where $m$ denote to the angular momentum quantum number, which take their values in the interval $-l \leq m \leq+l$, thus we have $(2 l+1)$ values for the orbital angular momentum quantum numbers.

\section{THE EXACT GLOBAL SPECTRUM OF THE LOWEST EXCITATIONS STATES FOR (M.C.P.) POTENTIAL IN (NC:3D- RSP) SYM- METRIES FOR QUARKOUNIOM SYSTEMS :}

Let us now resume the eigenenergies of the (d.s.e) obtained in this paper, the total modified energies $\left(E_{n c 0-c p}(\Theta, \bar{\theta}, \chi, \bar{\sigma}, m,, j, l, s, \delta, a, b)\right.$,

$\left.E_{n c 1-c p}(\Theta, \bar{\theta}, \chi, \bar{\sigma}, m, j, l, s, \delta, a, b)\right)$ of a quarkonium systems for ground states and first excited states produced with:

Ordinary three dimensional Cornell potential $V(r)=a r-\frac{b}{r}$, produce the values $E_{0 l}$ and $E_{1 l}$,

- Spin-orbital Hamiltonian operator $\hat{H}_{c p-p e r t}(r, A, B, \Theta, \bar{\theta})$ Producer $E_{n c 0-p e r}(\Theta, \bar{\theta}, a, b, \delta)$ and $E_{n c 1-p e r}(\Theta, \bar{\theta}, a, b, \delta)$,

- The modified Hamiltonian of Zeeman Effect: $\hat{H}_{m-c p}(r, A, B, \chi, \bar{\sigma}) \quad$ Producer $\quad E_{\text {mag0-cp }}(\chi, \bar{\sigma}, m, \delta, a, b)$ and $E_{\text {mag-1cp }}(\chi, \bar{\sigma}, m, \delta, a, b)$.

Which allow us to getting the global spectrum $E_{n c 0-c p}(\Theta, \bar{\theta}, \chi, \bar{\sigma}, m,, j, l, s, \delta, a, b)$ and $E_{n c 1-c p}(\Theta, \bar{\theta}, \chi, \bar{\sigma}, m, j, l, s, \delta, a, b)$ of the lowest excitations states for (m.c.p.) potential in (NC: 3D- RSP) symmetries for quarkonium systems: 


$$
\begin{aligned}
& E_{n c 0-c p}(\Theta, \bar{\theta}, \chi, \bar{\sigma}, m,, j, l, s, \delta, a, b)= \\
& =\frac{3 a}{\delta}-\frac{2 \mu\left(b+\frac{3 a}{\delta^{2}}\right)^{2}}{\left[ \pm \sqrt{1+4 l(l+1)+\frac{8 \mu a}{\delta^{3}}}\right]^{2}}+ \\
& \frac{1}{2} \alpha\left|N_{0 k}\right|^{2}\{j(j+1)+l(l+1)-s(s+1)\} \times \\
& \times\left(\Theta L_{o}(a, b, \delta)+\frac{\bar{\theta}}{2 m_{0}} T_{3}(a, b, \delta)\right)+ \\
& +\frac{1}{2} \alpha\left|N_{0 k}\right|^{2} m\left(\chi L_{o}(a, b, \delta)+\frac{\bar{\sigma}}{2 m_{0}} T_{3}(a, b, \delta)\right) H \\
& E_{n c 1-c p}(\Theta, \bar{\theta}, \chi, \bar{\sigma}, m,, j, l, s, \delta, a, b)= \\
& =\frac{3 a}{\delta}-\frac{2 \mu\left(b+\frac{3 a}{\delta^{2}}\right)^{2}}{\left[3 \pm \sqrt{1+4 l(l+1)+\frac{8 \mu a}{\delta^{3}}}\right]^{2}}+ \\
& +\frac{1}{2} \alpha\left|N_{1 k}\right|^{2} m\left(\chi L_{1-s}(a, b, \delta)+\frac{\bar{\sigma}}{2 m_{0}} L_{1-p}(a, b, \delta)\right) H \\
& \frac{1}{2} \alpha\left|N_{1 k}\right|^{2}\{j(j+1)+l(l+1)-s(s+1)\} \times \\
& \times\left(\Theta L_{1-s}(a, b, \delta)+\frac{\bar{\theta}}{2 m_{0}} T_{1-p}(a, b, \delta)\right)+
\end{aligned}
$$

In this way, one can obtain the complete energy spectra for (m.c.p.) potential in (NC: $3 D-R S P)$ symmetries. We have also the following accompanying constraint relations:

- The original spectrum for ground and first excited states contain two possible values of energies in ordinary three dimensional space which presented by equations (11) and (12),

- The quantum number $m$ satisfied the interval: $-l \leq m \leq+l$, thus we have $(2 l+1)$ values for this quantum number,

- The global momentum $j$ in the interval $|l-s| \leq j \leq|l+s|$ can be takes N values: $j=\underbrace{|l-s|,|l-s|+1, \ldots, 0, \ldots,|l+s|}$.

Allow us to deduce the important original results: every state in usually three dimensional space will be replace by $2(2 l+1) N$ sub-states and then the degenerated state can be take $2 N \sum_{i=0}^{n-1}(2 l+1) \equiv 2 N n^{2}$ values in (NC: 3D-RSP) symmetries . It's clearly, that the obtained eigenvalues of energies are real and then the noncommutative Hamiltonian operator $\hat{H}_{n c-c p}$ :

$$
\begin{aligned}
& \hat{H}_{n c-c p}=-\frac{1}{2 \mu}\left[\frac{1}{r^{2}} \frac{\partial}{\partial r}\left(r^{2} \frac{\partial}{\partial r}\right)+\frac{1}{r^{2} \sin \theta} \frac{\partial}{\partial \theta}\left(\sin \theta \frac{\partial}{\partial \theta}\right)+\right. \\
& \left.+\frac{1}{r^{2}(\sin \theta)^{2}} \frac{\partial^{2}}{\partial \phi^{2}}\right]+a r-\frac{b}{r}+\left\{\frac{\bar{\theta}}{2 \mu}+\Theta\left(\frac{b}{2 r^{3}}-\frac{a}{2 r}\right)\right\} \overleftrightarrow{S} \overleftrightarrow{L}+ \\
& +\left(\chi\left(\frac{b}{2 r^{3}}-\frac{a}{2 r}\right)+\frac{\bar{\sigma}}{2 \mu}\right)(\vec{H} \vec{J}-\overleftrightarrow{S} \overleftrightarrow{H})
\end{aligned}
$$

Which allows us to obtain the original results for this investigation: the obtained Hamiltonian operator (44) can be describing Quarkouniom systems: $c \bar{c}, b \bar{b}$, $c \bar{b}, b \bar{c}, \ldots .$. which has two permanent dipoles:

- The first is electric dipole moment,

- The second is magnetic moment in external stationary electromagnetic field.

It is important to notice that, the appearance of the polarization states of a fermionic particle for (m.c.p.) indicate to the validity of obtained results at very high energy where the relativistic equation Dirac will be applied, which allowing to apply these results of various Nano-particles at Nano scales. Finally, we can trace the following two important relations:

$$
\begin{aligned}
& \hat{H}_{n c-c p} \psi^{(0)}(\vec{r}) \equiv \\
& \equiv E_{n c 0-c p}(\Theta, \bar{\theta}, \chi, \bar{\sigma}, m,, j, l, s, \delta, a, b) \psi^{(0)}(\vec{r}) \\
& \hat{H}_{n c-c p} \psi^{(1)}(\ddot{r}) \equiv \\
& \equiv E_{n c 1-c p}(\Theta, \bar{\theta}, \chi, \bar{\sigma}, m,, j, l, s, \delta, a, b) \psi^{(1)}(\vec{r})
\end{aligned}
$$

Thus, we can resume the obtained results as (Ordinary three dimensional spaces $\rightarrow$ (NC: 3D-RSP) symmetries:

$$
\begin{gathered}
E_{0 l} \rightarrow E_{n c 0-c p}(\Theta, \bar{\theta}, \chi, \bar{\sigma}, m,, j, l, s, \delta, a, b) \\
E_{1 l} \rightarrow E_{n c 1-c p}(\Theta, \bar{\theta}, \chi, \bar{\sigma}, m,, j, l, s, \delta, a, b) \\
\hat{H}_{c p}=-\frac{1}{2 \mu}\left[\frac{1}{r^{2}} \frac{\partial}{\partial r}\left(r^{2} \frac{\partial}{\partial r}\right)+\frac{1}{r^{2} \sin \theta} \frac{\partial}{\partial \theta}\left(\sin \theta \frac{\partial}{\partial \theta}\right)+\right. \\
\left.+\frac{1}{r^{2}(\sin \theta)^{2}} \frac{\partial^{2}}{\partial \phi^{2}}\right]+a r-\frac{b}{r} \\
+\hat{H}_{n c-c p}=-\frac{1}{2 \mu}\left[\frac{1}{r^{2}} \frac{\partial}{\partial r}\left(r^{2} \frac{\partial}{\partial r}\right)+\frac{1}{r^{2} \sin \theta} \frac{\partial}{\partial \theta}\left(\sin \theta \frac{\partial}{\partial \theta}\right)+\right. \\
\left.+\frac{1}{r^{2}(\sin \theta)^{2}} \frac{\partial^{2}}{\partial \phi^{2}}\right]+a r-\frac{b}{r}+\left\{\frac{\bar{\theta}}{2 \mu}+\Theta\left(\frac{b}{2 r^{3}}-\frac{a}{2 r}\right)\right\} \overleftrightarrow{S} \overleftrightarrow{L}+ \\
+\left(\chi\left(\frac{b}{2 r^{3}}-\frac{a}{2 r}\right)+\frac{\bar{\sigma}}{2 \mu}\right)(\vec{H} \vec{J}-\overleftrightarrow{S} \overleftrightarrow{H})
\end{gathered}
$$

Our recent study gives a novel contributions corresponded the effect of noncommutativity of space phase compared with results of ref. [31]. It is easy to show that we recover the case of commutative space as a special case of noncommutative space and space phase, taking the parameters $\Theta, \bar{\theta}, \chi$ and $\bar{\sigma}$ equal zero.

\section{CONCLUSION}

In this study we have performed the exact analytical bound state solutions: the energy spectra and the corresponding noncommutative Hamiltonians for (d.s.e) with (m.c.p.) by using generalization Boopp's Shift method and standard perturbation theory. It is found that the energy eigenvalues depend on the dimensionality of the problem and new atomic quantum numbers 
$(j, l, s)$ and the angular momentum quantum number $m$ in addition to four infinitesimals parameters $(\Theta, \bar{\theta}, \chi, \bar{\sigma})$ and we also showed that the obtained energy spectra degenerate and every old state will be replaced by $2 N(2 l+1)$ sub-states. Finally, we expect that the results of our research are valid in the high energies, thus the (m.c.p) can gives the same results for

\section{REFERENCES}

1. Shi-Hai Dong, Guo-Hua Sun, Physica Scripta 70 No 2-3, 94 (2004).

2. J.J. Pena, G. Ovando, J. Morales, J. Phys.: Conf. Series 574, 012089 (2015).

3. L. Buragohain, S.A.S. Ahmed, Lat. Am. J. Phys. Educ. 4 No 1, 79 (2010).

4. A. Niknam, A. A. Rajab, M. Solaimani, J. Theor. Appl. Phys. 10, 53 (2016).

5. Sameer M. Ikhdair, Ramazan Sever, CEJP 5(4), 516 (2007).

6. M.M. Nieto, Am. J. Phys. 47, 1067 (1979).

7. S.M. Ikhdair, R. Sever, J. Mol. Struc.-Theochem. 806, 155 (2007).

8. A.S. Ahmed, L. Buragohain, Phys. Scr. 80, 1 (2009).

9. S.K. Bose, Nouvo Cimento B 113, 299 (1996).

10. G.P. Flesses, A. Watt, J. Phys. A: Math. Gen. 14, L315 (1981).

11. M. Ikhdair, R. Sever, Ann. Phys. (Leipzig) 16, 218 (2007).

12. S.H. Dong, Physica Scripta 64 No 4, 273 (2001).

13. S.H. Dong, Z.Q. Ma, J. Phys. A 31 No 49, 9855 (1998).

14. S.H. Dong, Int. J. Theor. Phys. 40 No 2, 559 (2001).

15. Ali Akder, J. Theor. Appl. Phys. 7, 17 (2013).

16. Sameer M. Ikhdair, Ramazan Sever, Adv. High Energ. Phys. 2013, Article ID 562959 (2013).

17. Shi-Hai Dong, Guo-Hua San, Found. Phys. Lett. 16 No 4, 357 (2003).

18. L. Buragohain, S.A.S. Ahmed, Lat. Am. J. Phys. Educ. 4 No 1,79 (2010).

19. S.M. Ikhdair, J. Modern Phys. 3 No 2, 170 (2012).

20. H. Hassanabadi, Tur. Phys. J. Plus. 127, 120 (2012).

21. H. Hassanabadi, M. Hamzavi, S. Zarrinkamar, A.A. Rajabi, Int. J. Phys. Sci. 6(3), 583 (2011).

22. Shi-Hai Dong, Zhoung-Qi Ma, Giampieero Esposito, Found. Phys. Lett. 12 No5 (1999).

23. D. Agboola, Acta Physica Polonica A 120, 371 (2011).

24. D. Shi-Hai, Int. J. Theor. Phys. 39, 1119 (2000).

25. Shi-Hai Dong, Physica Scripta, 64, 273 (2001).

26. B.I. Ita, Int. J. Recent Adv. Phys. (IJRAP) 2 No 4 (2013).

27. S.M. Kuchin, N.V. Maksimenko, J. Theor. Appl. Phys. 7, 47 (2013)

28. B.I. Ita, A.I. Ikeuba, A.N. Ikot, Commun. Theor. Phys. 61, 149 (2014).

29. S.M. Kuchin, N.V. Maksimenko, Universal J. Phys. Application. 1(3), 295 (2013).

30. H. Snyder, Phys. Rev. 71, 38 (1946).

31. A. Al-Jamel, J. Theor. Appl. Phys. 5-1, 21 (2011).

32. Abdelmadjid Maireche, The African Rev. Phys. 9: 0060, 479 (2014)

33. Abdelmadjid Maireche, J. Nano- Electron. Phys. 7 No 2, 02003 (2015)
Dirac and Klein-Gordon equations in the framework of the relativistic quantum mechanics.

\section{AKNOWLEDGEMENTS}

This work was supported with search laboratory of: Physique et Chimie des matériaux, in university of M'sila, Algeria.

34. Abdelmadjid Maireche, The African Rev. Phys. 9: 0025, 185 (2014)

35. Abdelmadjid Maireche, The African Rev. Phys. 10: 0014, 97 (2015).

36. Abdelmadjid Maireche, Int. Lett. Chem., Phys. Astronomy 56, 1 (2015).

37. Abdelmadjid Maireche, The African Rev. Phys. 10: 0046, 373 (2015).

38. Abdelmadjid Maireche, Med. J. Model. Simul. 04, 060 (2015).

39. Abdelmadjid. Maireche, Int. Lett. Chem., Phys. Astronomy 58, 164 (2015).

40. Abdelmadjid Maireche, Lat. Am. J. Phys. Educ. 09, 1301 (2015).

41. Abdelmadjid Maireche, Int. Lett. Chem., Phys. Astronomy 60, 11 (2015)

42. Abdelmadjid Maireche. J. Nano- Electron. Phys. 7 No 3, 02003 (2015).

43. Abdelmadjid Maireche, Int. Lett. Chem., Phys. Astronomy 61, 38 (2015).

44. Abdelmadjid Maireche, J. Nano- Electron. Phys. 7 No 4, 04021 (2015).

45. Abdelmadjid Maireche, The African Rev. Phys. 10, 177 (2015).

46. Abdelmadjid Maireche, Nano-World J. 1(4), 120 (2016).

47. Abdelmadjid Maireche, J. Nano- Electron. Phys. 8 No 1, 01020 (2016)

48. Abdelmadjid Maireche, J. Nano- Electron. Phys. 8 No 1, 01021 (2016)

49. A.E.F. Djemei, H. Smail, Commun. Theor. Phys. (Beijinig, China). 41, 837 (2004).

50. Shaohong Cai, Tao Jing, Guangjie Guo, Rukun Zhang, Int. J. Theoret. Phys. 49(8), 1699 (2010).

51. Joohan Lee, J. Korean Phys. Soc. 47 No 4, 571 (2005).

52. A. Jahan, Braz. J. Phys. 37 No 4, 144 (2007).

53. Anselme F. Dossa, Gabriel Y.H. Avossevou, J. Modern Phys. 4, 1400 (2013)

54. Yang, Zu-Hua, Int. J. Theor. Phys. 49, 644 (2010).

55. Y. Yuan, Chinese Phys. C 34(5), 543 (2010).

56. Jumakari-Mamat, Sayipjamal Dulat, Hekim Mamatabdulla, Int. J. Theor. Phys. 55, 2913 (2016)

57. Behrouz Mirza, Commun. Theor. Phys. 55, 405 (2011).

58. Yongjun Xiao, Zhengwen Long, Shaohong Cai, Int. J. Theor. Phys. 50, 3105 (2011).

59. A.E.F. Djemaï, H. Smail, Commun. Theor. Phys. 41, 837 (2004).

60. I.S. Gradshteyn, I.M. Ryzhik, Table of Integrals, Series and Products, 7th. ed. (Academic Press: Burlington, MA, USA: 2007). 